\title{
Scaling violation and shadowing corrections at HERA
}

\author{
A.L. Ayala Filho ${ }^{1,2, a}$, M.B. Gay Ducati ${ }^{2, b}$, E.M. Levin ${ }^{3,4, c}$ \\ 1 Instituto de Física, Univ. Federal do Rio Grande do Sul, Caixa Postal 15051, 91501-970 Porto Alegre, RS, Brazil \\ 2 Instituto de Física e Matemática, Univ. Federal de Pelotas, Campus Universitário, Caixa Postal 354, 96010-900, Pelotas, RS, \\ Brazil \\ 3 HEP Department, School of Physics and Astronomy, Raymond and Beverly Sackler Faculty of Exact Science, Tel Aviv \\ University, Tel Aviv 69978, Israel \\ 4 DESY Theory, Notkestrasse 85, 22607, Hamburg, Germany
}

Received: 21 September 1998 /Published online: 14 January 1999

\begin{abstract}
We study the value of shadowing corrections (SC) in the HERA kinematic region in the GlauberMueller approach. Since the Glauber-Mueller approach was proven in perturbative quantum chromodynamics (QCD) in the double-logarithmic approximation (DLA), we develop the DLA approach for the deep inelastic structure function which takes into account the SC. Our estimates show small SC for $F_{2}$ in the HERA kinematic region while they turn out to be sizable for the gluon structure function. We compare our estimates with those for gluon distribution in leading order (LO) and next to leading order (NLO) in the DGLAP evolution equations.
\end{abstract}

\section{Introduction}

We investigate the role of the shadowing corrections (SC) on the value of the deep inelastic structure functions and on the scaling violation mechanism in the HERA kinematic region, estimating the SC in the Glauber-Mueller approach proposed in [1-4].

The experimental results from the $\mathrm{e}\left(\mathrm{e}^{+}\right)$collider HERA present a steep increase of the proton structure function $F_{2}\left(x, Q^{2}\right)$ in the region of small values of the momentum fraction $x$. These data also show a strong scaling violation, i.e. a strong dependence of $F_{2}$ on the momentum transfer $Q^{2}$. In principle, all these experimental features can be described in the context of the perturbative quantum chromodynamics (pQCD) by the DGLAP evolution equations. In this approach, the small $x$ behaviour of the parton distributions inside the proton is driven by the gluon distribution. The number of quarks and antiquarks is generated by the perturbative QCD transition $g \rightarrow q \bar{q}$, which is the QCD mechanism to describe the scaling violation in $F_{2}$. Thus, the increase of the gluon distribution in the usual DGLAP evolution generates the strong rise of $F_{2}$.

Although DGLAP evolution equations can describe the data, new dynamical effects such as the parton interaction and recombination, which could modify the standard evolution, are not included in this parton evolution description. Indeed, as was shown in [2], QCD unitarization

\footnotetext{
a ayala@ufpel.tche.br

b gay@if.ufrgs.br

c leving@post.tau.ac.il
}

effects are expected to take place in the small $x$ region. For instance, the growth of the gluon distribution brings system to the high-density regime, even in the kinematic region where $\alpha_{\mathrm{S}}$ is small (large $Q^{2}$ region).

\section{The Glauber-Mueller approach for $F_{2}$}

A general approach to describe unitarization effects in QCD was proposed in [1]-[4]. In this approach, the SC are described in a Glauber model in QCD. The first step was the proof of a Glauber formula for QCD in the doublelogarithm approximation (DLA) by Mueller [1]. After that, the $s$-channel unitarity constraint was taken into account in the target rest frame to include unitarity effects in the high-energy scattering. From this approach, it was possible to obtain the Mueller formula and to propose a general eikonal approach to describe the SC.

In this eikonal approach, the deep inelastic scattering is described as follows. The high-energy virtual photon splits into a $q \bar{q}$ pair. This pair suffers multiscatterings within the target and the overall effect is the suppression of the $\gamma^{*}$-nucleon cross-section. Thus, the structure function $F_{2}\left(x, Q^{2}\right)$ is given by the eikonal expression [2-4]

$$
F_{2}\left(x, Q^{2}\right)=\frac{N_{\mathrm{c}}}{6 \pi^{3}} \sum^{N_{\mathrm{f}}} Z_{\mathrm{f}}^{2} \int_{\frac{1}{Q^{2}}}^{\infty} \frac{\mathrm{d} r_{\perp}^{2}}{r_{\perp}^{4}} \int \mathrm{d}^{2} b_{\perp}\left\{1-\mathrm{e}^{-\frac{1}{2} \Omega}\right\},
$$

where $Q^{2}$ is the photon virtuality, $N_{\mathrm{c}}$ is the number of colours and $Z_{\mathrm{f}}$ is the charge fraction of each quark. $N_{\mathrm{f}}$ is the number of flavours taken into account in the quarknucleon scattering and $b_{\perp}$ is the impact parameter for 


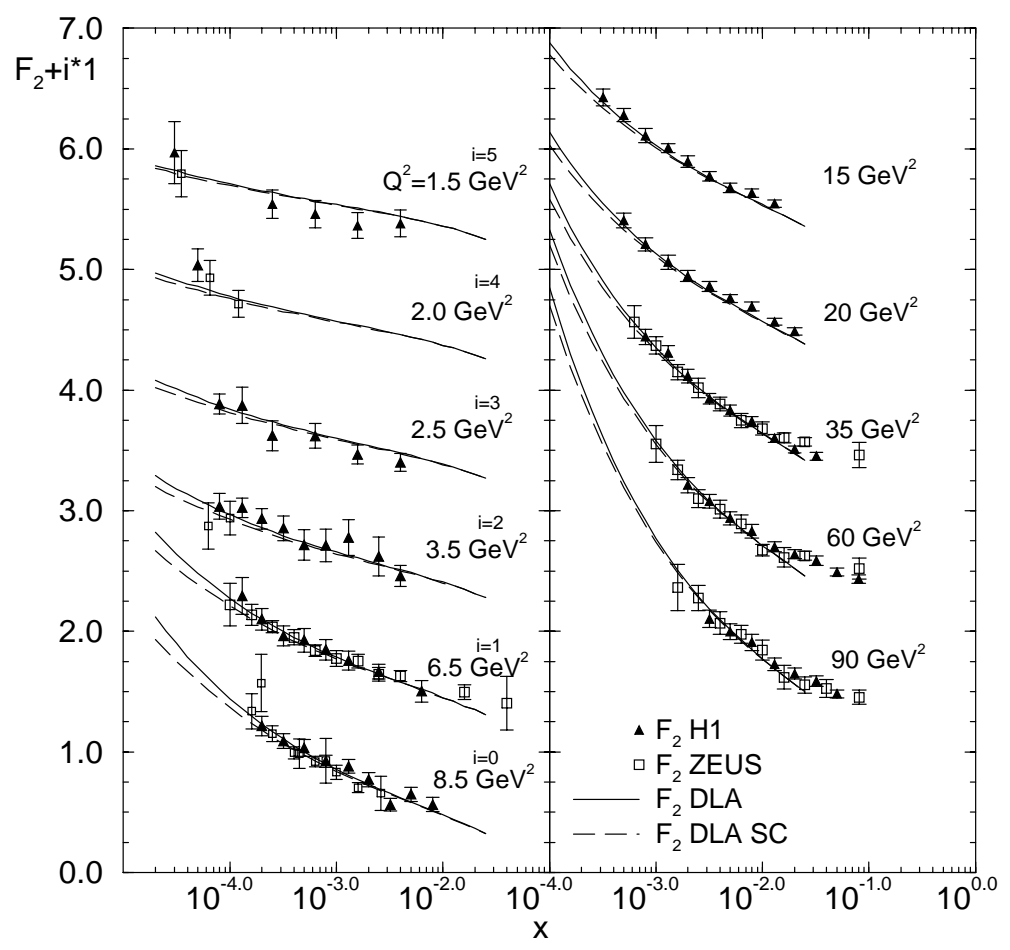

Fig. 1. The evolution of the structure function $F_{2}$ from the scaling violation mechanism in the DLA as a function of $x$. The solid line represents the Born term and the dashed line includes the SC the scattering of the quark-antiquark pair with transverse splitting $r_{\perp}$ with the nucleon target. The opacity function $\Omega$ is, generally speaking, an arbitrary real function and should be determined by a QCD-suggested model.

To construct a QCD model for $\Omega$, we took the DLA limit of perturbative QCD, i.e. the small $x$ and large $Q^{2}$ region, where we expect $\Omega$ to be small. In this region, we impose that (1) should reproduce the DGLAP evolution in the same limit. In [1] and [5], it was shown that the impact parameter dependence can be factorized out. Combining both results, $\Omega$ can be written as

$$
\Omega\left(x, Q^{2}, b_{\perp}\right)=\frac{4 \pi^{2} \alpha_{\mathrm{S}}}{3 Q^{2}} x G\left(x, Q^{2}\right) S\left(b_{\perp}\right),
$$

where $S\left(b_{\perp}\right)$ is the profile function for a nucleon with radius $R$, and is taken as an exponential function, $S\left(b_{\perp}\right)=$ $\left(1 / \pi R^{2}\right) \exp \left(-b_{\perp}^{2} / R^{2}\right)$, in our calculations. It should be stressed that the above procedure to obtain (1), with $\Omega$ defined by (2), takes into account the unitarity constraint in the DLA limit of pQCD and gives the DGLAP evolution equation [6] in the kinematic region where $\Omega \ll 1$. In order to investigate the $x$ and $Q^{2}$ evolution of the $F_{2}$ structure function in this approach, in [4] the gluon distribution was taken from the GRV95 set, which is a solution of the full DGLAP equation. Here, we are interested in developing a completely DLA formula and to compare this result with the ones obtained from the leading order (LO) and next to leading order (NLO) gluon distribution. Thus, we first develop a completely consistent DLA limit, where $F_{2}$ is related, through the scaling violation mechanism, to the DLA gluon distribution. We estimate the SC in this approach and, after that, compare the results with the SC predicted from LO and NLO gluon distribution. We then compare these results with the prediction of the Glauber formula for the gluon distribution.

Let us investigate first the role of SC in the completely consistent DLA limit. We consider the Born term of (1) with the gluon distribution taken in the DLA limit. Since the Born term is equivalent to the DGLAP expression for $F_{2}$ in the DLA limit, we will take $x G^{\mathrm{DLA}}$ as in [7] and [8]. Thus, the gluon distribution reads

$$
x G^{\mathrm{DLA}}\left(x, Q^{2}\right)=G_{0} I_{0}(y),
$$

where the variables are $y=2 \gamma \sqrt{\ln (1 / x) \ln \left(t / t_{0}\right)}, t=$ $\ln \left(Q^{2} / \Lambda^{2}\right)$ and $t_{0}=\ln \left(Q_{0}^{2} / \Lambda^{2}\right)$. The QCD constants are $\gamma=\sqrt{12 / \beta_{0}}, \beta_{0}=11-\left(2 N_{\mathrm{f}} / 3\right)$ and $\Lambda=0.232 \mathrm{GeV}^{2}$. The constant $G_{0}$ plays the role of the flat initial condition, since the Bessel function $I_{0}(y)$ goes to 1 as $y$ goes to zero. We disregard the sub-leading corrections to the DLA gluon proportional to $\alpha_{\mathrm{S}} \ln Q^{2}$ proposed in [7]. If we integrate (1) over $b_{\perp}$ the Born term then reads

$$
F_{2}=F_{2}\left(x, Q_{0}^{2}\right)+\frac{2}{9 \pi} \int_{Q_{0}^{2}}^{Q^{2}} \frac{\mathrm{d} Q^{\prime 2}}{Q^{\prime 2}} \alpha_{\mathrm{S}} x G^{\mathrm{DLA}}\left(x, Q^{\prime 2}\right),
$$

where we have taken $N_{\mathrm{f}}=3$ and $\alpha_{\mathrm{S}}$ as a series. The expression (4) gives the sea component of $F_{2}$ generated by the gluon evolution from the initial virtuality $Q_{0}^{2}$ up to $Q^{2}$. Going from expression (1) to (4) we have taken $Q^{\prime 2}=1 / r_{\perp}^{2}$. Thus, the lower limit $Q_{0}^{2}$ works as a cut off for the large distance effects over $F_{2}$. These effects are included in $F_{2}\left(x, Q_{0}^{2}\right)$, the value of the structure function for the virtuality $Q_{0}^{2}$. It has a nonperturbative origin and takes into account the number of $\mathrm{q} \overline{\mathrm{q}}$ pairs not generated by the perturbative transition $\mathrm{g} \rightarrow \mathrm{q} \overline{\mathrm{q}}$. We parametrize the initial structure function by the expression

$$
F_{2}\left(x, Q_{0}^{2}\right)=C_{0} x^{-0.08}(1-x)^{10},
$$



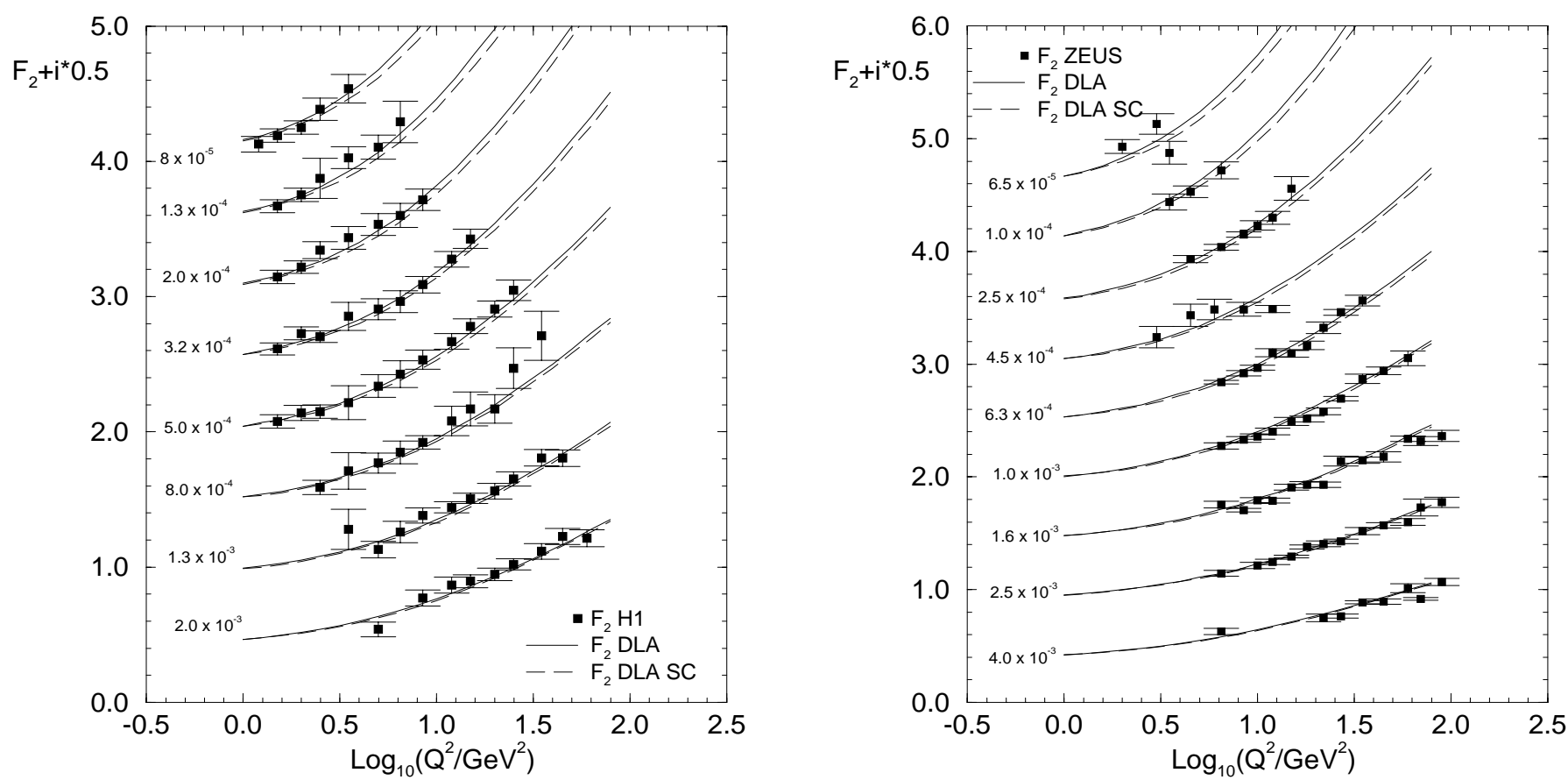

Fig. 2. $F_{2}$ evolution from the scaling violation mechanism in the DLA as a function of $Q^{2}$ (for the scaling violation figures, the value of $i$ goes from 0 for $x=2.0 \times 10^{-3}$ to 7 for $x=8.0 \times 10^{-5}$ )

where $C_{0}$ is a constant that adjusts the nonperturbative contribution. This expression reproduces the soft pomeron behaviour $\left(x^{-0.08}\right.$ as $\left.x \rightarrow 0\right)$ presented by the $\gamma^{*}$-nucleon cross-section in the low $Q^{2}$ region [9]. Since we have used $N_{\mathrm{f}}=3$, we should add the charm component $F_{2}^{\mathrm{c}}$. This component is generated perturbatively from the $\gamma^{*}$-gluon fusion mechanism with the gluon distribution given by the DLA expression (3). This mechanism is discussed in detail in [10]. Finally, we obtain the following expression for $F_{2}$

$$
F_{2}\left(x, Q^{2}\right)=F_{2}\left(x, Q_{0}^{2}\right)+F_{2, \text { DLA }}^{\text {Born }}+F_{2, D L A}^{\mathrm{c}} .
$$

To fit the expression (6) to the HERA data, we have taken the $F_{2}$ points which lie in the region $1 \mathrm{GeV}^{2}<\mathrm{Q}^{2}<$ $100 \mathrm{GeV}^{2}$ and $x<10^{-2}$, where we expect that our DLA approach of SC is valid. The $\mathrm{H} 1$ and ZEUS results were taken from [11] and [12], respectively.

In Fig. 1 we present the fit for a subset of the data. The parameters used are $G_{0}=0.136, C_{0}=0.273$ and $Q_{0}^{2}=0.330 \mathrm{GeV}^{2}$. The values of the parameters were chosen in such a way to minimize the $\chi^{2}$, which corresponds to $\chi^{2} /$ d.o.f. $=124 / 222$ (where d.o.f stands for degrees of freedom). We can see from the figure that the steep behaviour of the deep inelastic structure function is well described by the DLA evolution of the gluon distribution, provided we have included enough nonperturbative $q \bar{q}$ pairs. With this set of parameters the $Q^{2}$ scaling violation of $F_{2}$ can also be described, as shown in Fig. 2. Taking a small value for the initial virtuality we can generate the DLA behaviour for $Q^{2} \approx 2 \mathrm{GeV}^{2}$. A similar result was obtained in [8], but in an incomplete DLA limit ${ }^{1}$. It is important to note

\footnotetext{
1 The authors have taken $P_{\mathrm{qg}}=z^{2}+(1-z)^{2}$ and the sub-
} leading factor $\left(t / t_{0}\right)^{-\delta}$ with $\delta=\left(11+\left(2 N_{\mathrm{f}} / 27\right)\right) / \beta_{0}$. that our aim is to describe HERA data in a completely consistent DLA limit, and not to provide an overall fit to existing high-energy data.

Now, we are able to investigate the number of SC predicted for $F_{2}$ in the Glauber-Mueller approach in a consistent DLA limit. For that, we consider the eikonal expression (1) with the opacity $\Omega$ calculated from the DLA gluon distribution obtained above. In Fig. 1 we present the results for $F_{2}$ as a function of $x$, and in Fig. 2, as a function of $Q^{2}$. As we can see, the SC are important only for very small values of $x$ and moderate values of $Q^{2}$. We would like to recall that in (1) we put the upper limit of integration equal to $1 / Q_{0}^{2}$, or in other words we consider only the SC which originate from sufficiently short distances, namely, $r_{\perp} \leq 1 / Q_{0} \approx 0.35 \mathrm{fm}$. In fact, we do not take into account the SC at large distances considering that they have been included in the initial parton distribution of (5).

Therefore, we are calculating only perturbative shadowing. In the kinematic region of the present data, the corrections lie within the experimental error.

We plot also in Figs. 3 and 4 the SC for $F_{2}$ predicted by the Glauber approach taking into account the LO and NLO gluons. As, in both cases, the gluon distribution does not present the DLA behaviour in the accessible kinematic region (see [4] for details), we have used the modified Mueller formula discussed in [4]. In this formula, the Born term is taken in the leading $\alpha_{\mathrm{S}} \ln Q^{2}$ aproximation $\left(\operatorname{LLA}\left(Q^{2}\right)\right)$, while the correction term is taken in the DLA. For practical purposes, we use the structure function $F_{2}$, i.e. the solution of the DGLAP equations, as the Born term in expression (6). We have taken only the GRV distribution since those distributions evolve from small vir- 


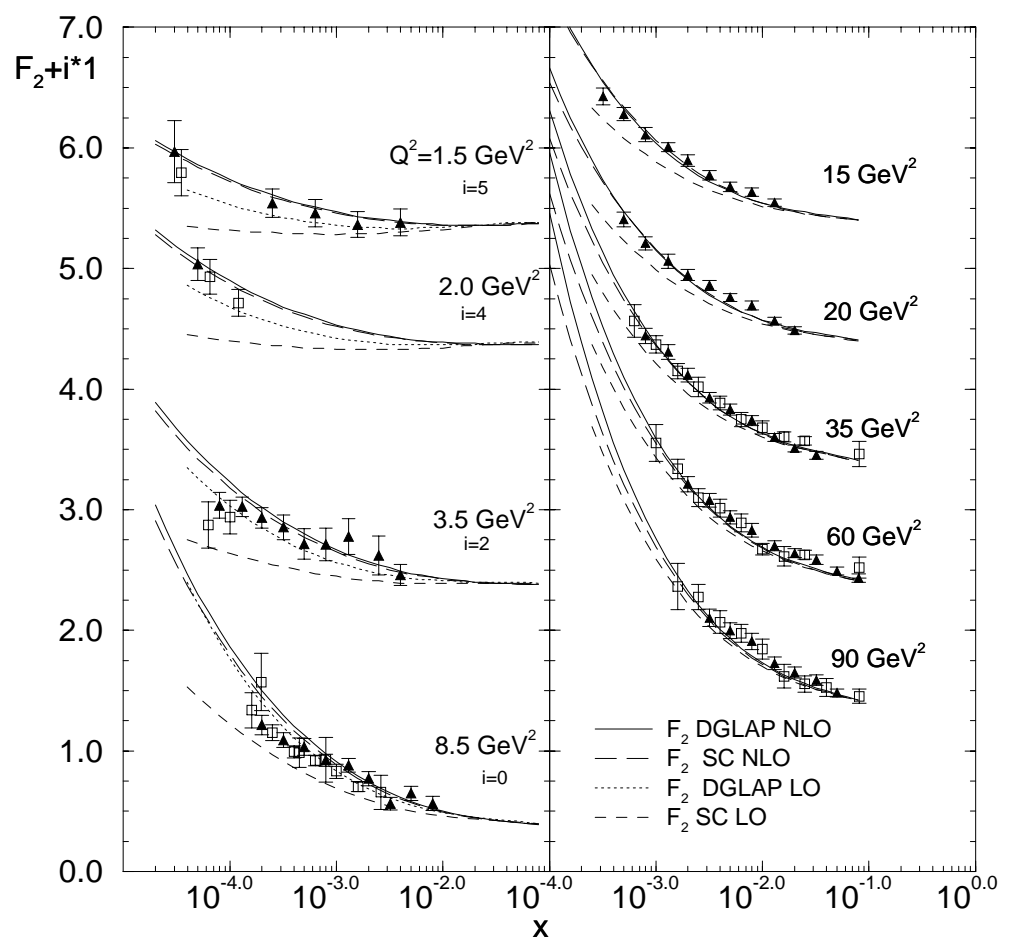

Fig. 3. The evolution of the structure function $F_{2}$ in $\mathrm{LO}$ and $\mathrm{NLO}$ as a function of $x$. The Born term (DGLAP evolution) for $F_{2}$ NLO and LO numerically coincide for $Q^{2}>10 \mathrm{GeV}^{2}$
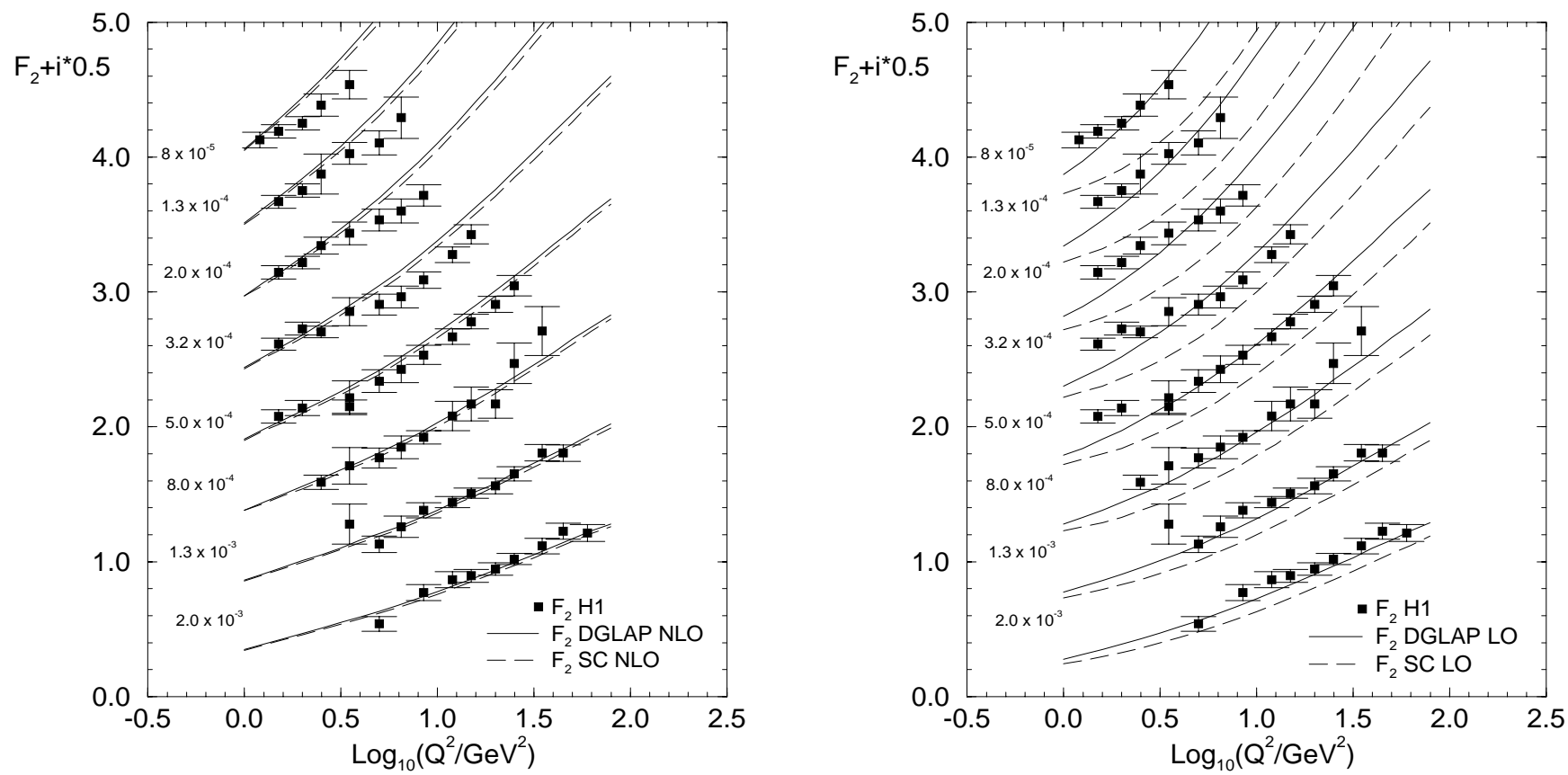

Fig. 4. $F_{2}$ evolution from the scaling violation mechanism in LO and NLO as a function of $Q^{2}$

tualities and can be compared with our DLA approach. We see from the figures that the LO gluon predicts much more SC to $F_{2}$. It means that the scaling violation suffers a stronger modification for the LO gluon than for the simple DLA gluon and the NLO gluon.

\section{Corrections to the Glauber-Mueller approach}

The eikonal approach for $F_{2}$ taken into account so far cannot be considered as a full description of the SC in deep inelastic scattering. That is because it was assumed that only a quark-antiquark pair embodies multi-rescatterings with the target and no SC were included in the gluon distribution. As was shown in [3], [4] and [13] the gluon rescatterings turn out to be essential. To investigate the 

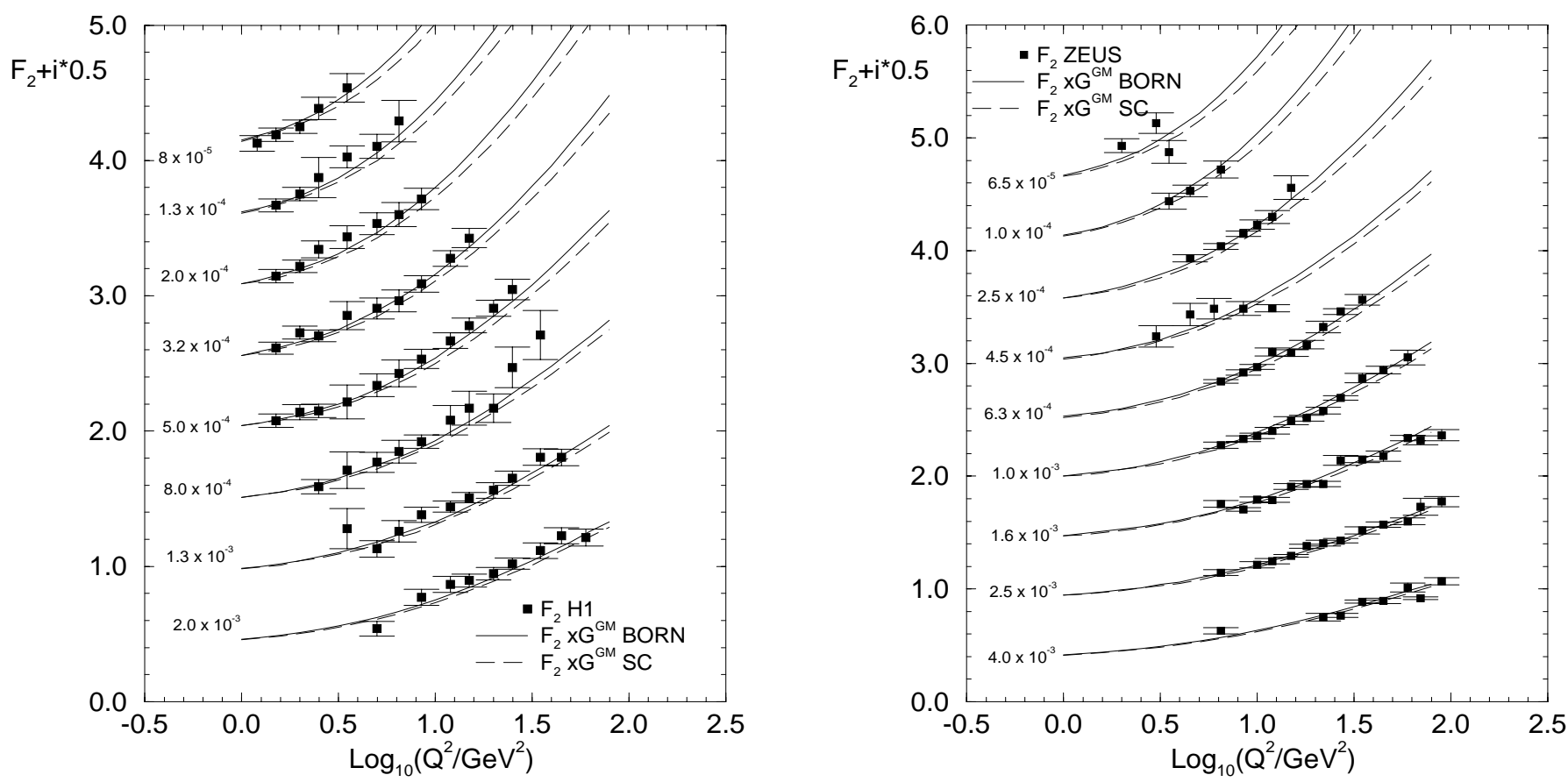

Fig. 5. $F_{2}$ evolution from the scaling violation mechanism in DLA with the gluon distribution $x G^{\mathrm{GM}}$ given by the GlauberMueller approach
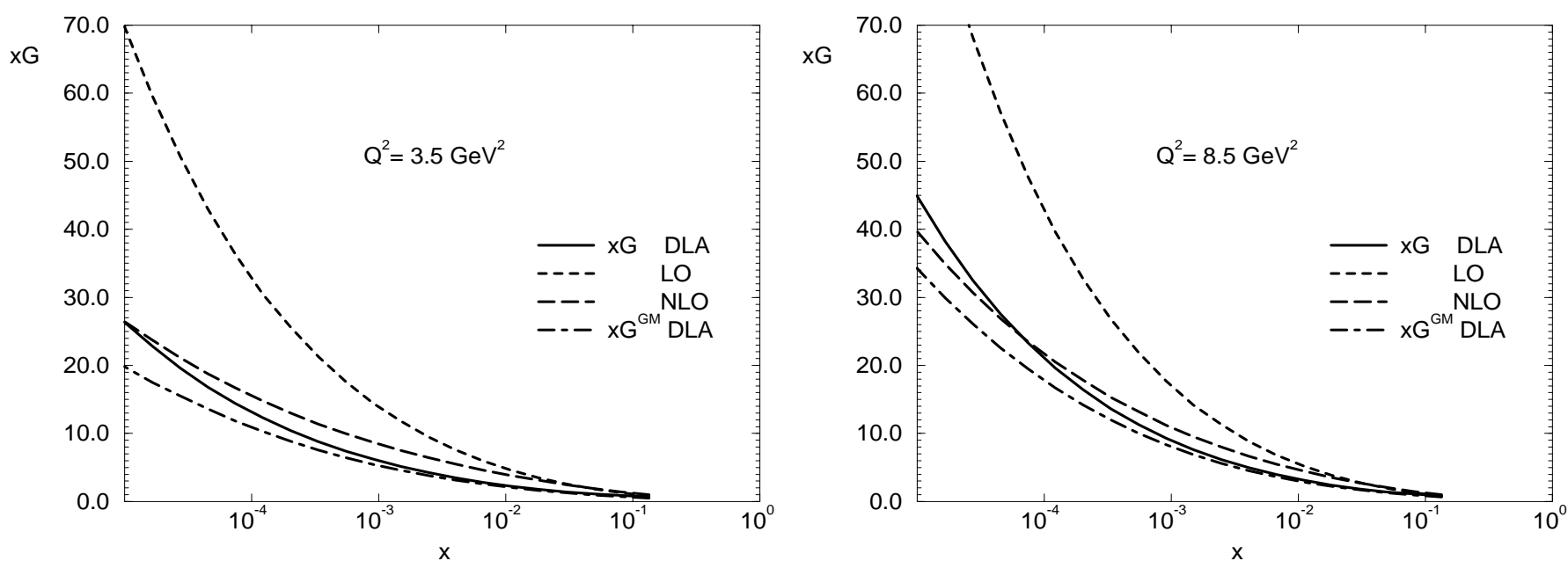

Fig. 6. The gluon distributions in DLA with and without SC calculated from the Glauber-Mueller approach, compared with LO and NLO gluon distributions from the GRV95 set

role of the gluon rescattering we calculate here the Born term of (1) but given by the expression

$$
F_{2}=F_{2}\left(x, Q_{0}^{2}\right)+\frac{2}{9 \pi} \int_{Q_{0}^{2}}^{Q^{2}} \frac{\mathrm{d} Q^{\prime 2}}{Q^{\prime 2}} \alpha_{\mathrm{S}} x G^{\mathrm{GM}}\left(x, Q^{\prime 2}\right),
$$

where $x G^{\mathrm{GM}}$ is the gluon structure function calculated in the eikonal (Glauber-Mueller) approach, namely

$$
\begin{aligned}
x G^{\mathrm{GM}}\left(x, Q^{2}\right)= & \frac{2}{\pi^{2}} \int_{\frac{1}{Q^{2}}}^{\frac{1}{Q_{0}^{2}}} \frac{\mathrm{d} r_{\perp}^{2}}{r_{\perp}^{4}} \int_{x}^{1} \frac{\mathrm{d} x^{\prime}}{x^{\prime}} \\
& \times \int_{0}^{\infty} \mathrm{d} b_{\perp}^{2}\left\{1-\mathrm{e}^{-\frac{\Omega_{\mathrm{G}}\left(x^{\prime}, r_{\perp}^{2}, b_{\perp}\right)}{2}}\right\},
\end{aligned}
$$

where the opacity $\Omega_{G}=\frac{9}{4} \Omega$. Expression (8) is the Mueller formula which was obtained in [4]. When (8) is included in expression (7), the Born term reproduces (4), since it is the DGLAP equation in the DLA limit. The other terms take into account the $\mathrm{SC}$ to the gluon distribution. The results are shown in Fig. 5. Comparing Figs. 2 and 5, one can see that the SC due to gluon rescattering are bigger than the corrections due to quark rescattering. However, both lie within the experimental error.

In order to complete our discussion, we plot in Fig. 6 the DLA gluon distribution given by expression (3) and the corrected gluon distribution given by the modified Mueller formula (8). The LO and NLO gluon distribution given by the parametrization GRV95 are also plotted. As 
we can see, the DLA distribution predicts a number of gluons closer to the NLO DGLAP evolution. This is not a coincidence, since both $x G^{\mathrm{DLA}}$ and the NLO GRV distribution have a flat behaviour for $Q^{2}=0.4 \mathrm{GeV}^{2}$ while the LO gluon distribution already has a steep behaviour in the small $x$ region for this low $Q^{2}$ value.

\section{Conclusions}

We can see from the results presented in Fig. 6 that the SC turn out to be sizable (about $23 \%$ for $Q^{2}=3.5 \mathrm{GeV}^{2}$ and $12 \%$ for $Q^{2}=8.5 \mathrm{GeV}^{2}$ ) in the gluon structure function but their manifestation in $F_{2}$ is rather small as we have already discussed (see Fig. 5). Comparing also Figs. 2, 4 and 5 we can see that the SC for $F_{2}$ have a strong dependence on the number of gluons taken into account in the QCD evolution. This is our main conclusion. It calls for new measurements in the high-energy kinematic region that are more sensitive to the value of the gluon structure function than the measurements of $F_{2}$.

Acknowledgements. ALAF acknowledges CAPES and MBGD acknowledges CNPq for partial financing.

\section{References}

1. A.H. Mueller: Nucl. Phys. B 335, 115 (1990)

2. A.L.Ayala, M.B.Gay Ducati, E.M. Levin: Phys. Lett. B 388, 188 (1996)

3. A.L.Ayala, M.B.Gay Ducati, E.M. Levin: Nucl. Phys. B 493, 305 (1997)

4. A.L.Ayala, M.B.Gay Ducati, E.M. Levin: Nucl. Phys. B 511, 355 (1998)

5. E.M. Levin, M.G. Ryskin: Sov. J. Nucl. Phys. 45 (1987) 150; B. Blättel, G. Baym, L.L Frankfurt, M. Strikman: Phys. Rev. Lett. 70, 896 (1993)

6. V.N. Gribov, L.N. Lipatov: Sov. J. Nucl. Phys. 15, 438 (1972); L.N. Lipatov: Yad. Fiz. 20, 181 (1974); G. Altarelli, G. Parisi: Nucl. Phys. B 126, 298 (1977); Yu.L. Dokshitzer: Sov. Phys.-JETP 46, 641 (1977)

7. R.D. Ball, S. Forte, Phys. Lett. B 335, 77 (1994)

8. Z. Huang, H. Jung Lu, I. Sarcevic: hep-ph/9705250 (1997)

9. A. Donnachie, P.V. Landshoff: Phys. Lett. B 244, 322 (1984), Z. Phys. C 61, 139 (1994)

10. M. Glück, E. Reya, A. Vogt: Z. Phys. C 67, 433 (1995)

11. H1 collaboration, S. Aid et al.: Nucl. Phys. B 470, 3 (1996); C. Adloff et al.: Nucl. Phys. B 497, 3 (1997)

12. ZEUS collaboration, M. Derrick et al.: Z. Phys. C 69, 607 (1996); M. Derrick et al.: Z. Phys. C 69, 399 (1996)

13. L. V. Gribov, E. M. Levin, M. G. Ryskin: Phys. Rep. 100, 1 (1983) 